\title{
Initial Velocity Effect on Acceleration Fall of a Spherical Particle through Still Fluid
}

\author{
Zegao Yin, Zhenlu Wang, Bingchen Liang, and Li Zhang \\ Engineering College, Ocean University of China, Qingdao, China \\ Correspondence should be addressed to Bingchen Liang; bingchen@ouc.edu.cn
}

Received 10 October 2016; Accepted 4 January 2017; Published 6 February 2017

Academic Editor: Sébastien Poncet

Copyright (C) 2017 Zegao Yin et al. This is an open access article distributed under the Creative Commons Attribution License, which permits unrestricted use, distribution, and reproduction in any medium, provided the original work is properly cited.

\begin{abstract}
A spherical particle's acceleration fall through still fluid was investigated analytically and experimentally using the BassetBoussinesq-Oseen equation. The relationship between drag coefficient and Reynolds number was studied, and various parameters in the drag coefficient equation were obtained with respect to the small, medium, and large Reynolds number zones. Next, some equations were used to derive the finite fall time and distance equations in terms of certain assumptions. A simple experiment was conducted to measure the fall time and distance for a spherical particle falling through still water. Sets of experimental data were used to validate the relationship between fall velocity, time, and distance. Finally, the initial velocity effect on the total fall time and distance was discussed with different terminal Reynolds numbers, and it was determined that the initial velocity plays a more important role in the falling motion for small terminal Reynolds numbers than for large terminal Reynolds number scenarios.
\end{abstract}

\section{Introduction}

Since the middle of the 19th century, a solid particle falling in gases or liquids has been investigated in many fields, such as hydraulics and chemistry. Considerable attention has been given to the steady-state motion of a spherical particle falling in incompressible Newtonian fluids, especially for the relationship between drag coefficient $C_{D}$ and Reynolds number $R$. Stokes [1] neglected the inertia item in the NavierStokes equations and proposed an inversely proportional relationship between $C_{D}$ and $R$ with $R<0.1$ scenarios for the first time. Then, the inertia item was taken into account by Oseen $[2,3]$, and an improved relationship was proposed for $R<0.4$ scenarios. Then, the perturbation theory was used to obtain a $C_{D}$ formula for $R<1$ scenarios $[4,5]$. A more recent study on $C_{D}$ correlations with $R$ can be found in the works of Chester et al., Clift et al., and Liao and Julien [6-9]. It should be noted that most of these aforementioned correlations had little deviations [10].

In contrast to the steady-state fall of a particle, an unsteady-state fall has an acceleration process before it reaches the terminal fall velocity. Chang and Yen [11] discussed the importance of added mass and the Basset force of a sphere falling for a low sphere-to-fluid density ratio and low
$R$. Jalaal et al. [12] used the homotopy perturbation method to derive the fall velocity, acceleration, and distance equations of a spherical particle falling unsteadily in a Newtonian fluid, ignoring the effect of the Basset force. Guo [13] analysed the acceleration fall behaviour of a sphere through still fluid for arbitrary Reynolds numbers and presented a simple solution for fall distance and velocity. Yin et al. [14] neglected the Basset force effect and experimentally investigated a spherical particle acceleration fall through still water with large $R$.

The objective of our study was to investigate the initial velocity effect on a spherical particle acceleration fall through still fluid analytically and experimentally. In this study, the relationship between $C_{D}$ and $R$ was investigated, and different parameters in the $C_{D}$ equation were obtained with respect to small, medium, and large $R$ zones. Then, the finite fall time and distance equations were derived in terms of some assumptions by using the results reported by Guo [13]. Furthermore, a simple experiment was conducted to measure the fall time and distance for a spherical particle falling through still water. In addition, sets of experimental data were used to validate the relationship between fall velocity, time, and distance. The initial velocity effect on fall time and distance was discussed with different terminal Reynolds numbers. Finally, the conclusions are summarized. 


\section{Kinetic Equation of an Acceleration Fall}

2.1. Equation Derivations. Concerning a spherical particle falling with acceleration through still fluid, the classic BassetBoussinesq-Oseen (BBO) equation has often been used to depict the behaviour of the fall $[3,11-13,15-17]$.

$$
\begin{aligned}
m_{s} \frac{d w}{d t}= & \left(m_{s}-m\right) g-\frac{C_{D}}{2} \rho w^{2}\left(\frac{\pi D^{2}}{4}\right)-C_{A 0} m \frac{d w}{d t} \\
& -C_{B} D^{2}(\pi \rho \mu)^{1 / 2} \int_{0}^{t} \frac{d w}{d \tau} \frac{d \tau}{(t-\tau)^{1 / 2}},
\end{aligned}
$$

where the left-hand side of (1) is the inertia of a spherical particle. The first, second, third, and fourth terms on the right-hand side are its submerged weight, drag, added mass force, and Basset history force, respectively. $m_{s}$ is the spherical particle mass, $w$ is the fall velocity, $t$ is the time, $m$ is the mass of fluid for the same volume of spherical particle, $g$ is the gravitational acceleration, $\rho$ is the fluid density, $D$ is the diameter of spherical particle, $C_{D}$ is the drag coefficient, $C_{A 0}$ is the classic added mass coefficient, $C_{B}$ is the classic Basset force coefficient, $C_{B}=3 / 2, \mu$ is the dynamic viscosity of fluid, and $\tau$ is the dummy variable for integration.

The submerged weight is given by subtracting the buoyancy force from the particle weight, and it is well accepted that this term plays an important role in the total force [9]. The contribution of drag force is decided by $C_{D}$ to a great extent. Considerable attention has been given to its value with different $R(R=w D / \nu)$, in which $\nu$ is the kinematic viscosity of fluid $[1-3,7,11-13]$. In terms of $R$, it can be classified as small, medium, and large numbers, and the corresponding critical values are assumed to be $R_{\mathrm{cr} 1}=$ $w_{\mathrm{cr} 1} D / v=0.1$ and $R_{\mathrm{cr} 2}=w_{\mathrm{cr} 2} D / \nu=1000[13,18]$, where $w_{\text {cr1 }}=0.1 \nu / D$ is defined as the first critical velocity between small and medium $R$ zones, $w_{\mathrm{cr} 2}=1000 v / D$ is defined as the second critical value between medium and large $R$ zones, and their corresponding times were defined as $t_{\mathrm{cr} 1}$ and $t_{\mathrm{cr} 2}$, respectively. The relationship between $C_{D}$ and $R$ can be expressed as the following general expression with Rubey's drag law [19]:

$$
C_{D}=\frac{\beta}{R}+\alpha
$$

For small $R$ in a laminar zone $(R<0.1), \alpha=0$ and $\beta=24$ [1]. For large $R$ in a turbulent zone $\left(1000<R<10^{5}\right)$, $\alpha=0.44 \pm 0.06$ and $\beta=24$, which agreed well with the experimental data over a wide range of Reynolds numbers [13]. As for medium $R$ in a transitional zone $(0.1<R<$ 1000), Lapple and Shepherd [20] stated that $C_{D}$ decreases with increasing $R$ :

$$
C_{D}=\frac{24\left(1+0.125 R^{0.72}\right)}{R} .
$$

The range of $C_{D}$ deviation in (3) is $-8 \%$ to $5 \%$ [7]. Using (3), results for $R$ range from 0.1 to 1000 at a step of 0.1 were obtained, and (3) was fitted as

$$
C_{D}=\frac{25.057}{R}+0.5
$$

The correlation coefficient is 0.997. Therefore, (4) can be used to depict the relationship between $C_{D}$ and $R$ in a transitional zone without causing major error.

Concerning the added mass force term, it deserves a brief description. One important property of the solid-fluid interaction is that the solid is not entirely free to move within the fluid. As the solid particle moves from one position to the other, an equal volume of fluid must move in the opposite direction. So if the mass of solid particle is accelerated, the mass of an equal volume of fluid must also be accelerated. And this concept is referred to as the added mass [9]. In (1), $C_{A 0}$ is equal to $1 / 2$ for the creeping motion after the integration of the potential unsteady pressure over the sphere's surface [21]. Hamilton and Lindell [22] also concluded that $C_{A 0}$ is consistently experimentally close to 0.5 for $R$ values up to 35000 , by neglecting the sphere acceleration effect. In contrast, $C_{A 0}$ increases with $R$ because of flow separation [9]. Odar [23] further correlated $C_{A 0}$ using an instantaneous acceleration modulus; nevertheless, the modulus is arduous to obtain explicitly.

The Basset force contribution to total force was determined by the density ratio $\rho / \rho_{s}$ to a considerable extent, where $\rho_{s}$ is the density of the particle. It was found that the Basset force is always important in the calculations for $\rho / \rho_{s}>1$ scenarios. For some heavy particles in air such as $\rho / \rho_{s}<0.004$, the Basset force plays an insignificant role in the total force. The Basset force contribution for $0.004<\rho / \rho_{s}<$ 1 depends on the type of particles and on the frequency of the fluid velocity fluctuations [24]. Particular attention has been devoted to the importance of the Basset force compared to the other hydrodynamic forces [25]. Lawrence and Weinbaum [26] investigated the unsteady force on a sphere at low Reynolds number and pointed out that the Basset force for an arbitrary velocity contained a new memory integral whose kernel differed from the classical behaviour derived by Basset [15]. Sobral et al. [27] considered the unsteady motion of a rigid spherical particle in fluid flows and found that its motion is significantly affected by the Basset force on the early stages of the motion and on the approach to the steady state. Due mainly to the complexity of the Basset force, a series of numerical methods were employed to resolve it, such as the Laplace transform method [28], the "window model" [29], and fractional-derivative approach [30]. However, its computation was far more expensive than other items in the BBO equation because of the dramatic demands of computational time and memory. In contrast to the numerical solutions, its analytical solution has advantages including simplicity and reliability. However, the analytic solutions of a spherical particle falling in still water were still not found. Attempts to resolve this dilemma were made for the theoretical solution of the Basset force and BBO equation with a simplified method. Guo [13] assumed $d w / d \tau$ 
to be a constant to separate it from the definite integral and $D^{2}(\pi \rho \mu)^{1 / 2} \int_{0}^{t}\left(d \tau /(t-\tau)^{1 / 2}\right)$ as $m$ in the Basset force item. He empirically combined the added mass force and Basset force into one integrated term, $C_{A} m(d w / d t)\left(C_{A}\right.$ is the integrated added mass coefficient), and the fall velocity was expressed as

$$
w=\frac{\left(2 c-b w_{0}\right)+k w_{0} \operatorname{coth}\left(k\left(t-t_{0}\right) / 2\right)}{\left(b+2 a w_{0}\right)+k \operatorname{coth}\left(k\left(t-t_{0}\right) / 2\right)},
$$

where $a=(3 \alpha / 4)\left(1 /\left(\Delta+C_{A}\right) D\right), b=(3 \beta / 4)\left(v /\left(\Delta+C_{A}\right) D^{2}\right)$, $c=(\Delta-1) g /\left(\Delta+C_{A}\right), k=\sqrt{b^{2}+4 a c}, \Delta=\rho_{s} / \rho, t_{0}$ is the initial time, and $w_{0}$ is the initial velocity. The terminal fall velocity, $w_{T}$, can be deduced with (5) for $t \rightarrow \infty$ :

$$
w_{T}=\frac{(k-b)}{(2 a)} .
$$

Using (5) with $w_{\mathrm{cr} 1}=0.1 v / D$ or $w_{\mathrm{cr} 2}=1000 \nu / D, t_{\mathrm{cr} 1}$ and $t_{\mathrm{cr} 2}$ can be expressed as

$$
\begin{aligned}
& t_{\mathrm{cr} 1}=\frac{1}{k} \ln \frac{(b v+0.2 a D+k v)\left(b+2 a w_{0}-k\right)}{(b v+0.2 a D-k v)\left(b+2 a w_{0}+k\right)}+t_{0} \\
& t_{\mathrm{cr} 2}=\frac{1}{k} \ln \frac{(b v+2000 a D+k v)\left(b+2 a w_{0}-k\right)}{(b v+2000 a D-k v)\left(b+2 a w_{0}+k\right)}+t_{0} .
\end{aligned}
$$

The fall distance was expressed as

$$
\begin{aligned}
s= & \frac{1}{a} \ln \frac{\left(k-b-2 a w_{0}\right) e^{-k\left(t-t_{0}\right)}+\left(k+b+2 a w_{0}\right)}{2 k} \\
& +\frac{(k-b)\left(t-t_{0}\right)}{2 a} .
\end{aligned}
$$

Assuming an initial fall velocity of zero, Guo [13] derived the following relationship between fall distance and fall velocity:

$$
s=\frac{1}{2 a}\left(\ln \frac{c}{c-b w-a w^{2}}-\frac{2 b}{k} \tanh ^{-1} \frac{k w}{2 c-b w}\right),
$$

where $\tanh ^{-1}$ is the inverse hyperbolic function.

2.2. Finite Values of Acceleration Fall Time and Distance. For $t \rightarrow \infty$, the fall distance in (8) approaches an infinite value. In order to simplify the study, the finite values of fall time and distance were expressed with the following assumptions.

For $w_{0}<0.99 w_{T}$ scenarios, the acceleration fall process was assumed to be completed when the fall velocity reached $99 \%$ of the terminal fall velocity. Applying (6) and $w=$ $0.99 w_{T}$ to (5) gives the corresponding time as follows:

$$
t_{S}=\frac{1}{k} \ln \frac{(1.99 k+0.01 b)\left(b+2 a w_{0}-k\right)}{(0.01 b-0.01 k)\left(b+2 a w_{0}+k\right)}+t_{0} .
$$

Substituting (10) into (8) gives

$$
s_{S}=\frac{k+b}{2 a} \ln \frac{b+2 a w_{0}+k}{1.99 k+0.01 b}+\frac{k-b}{2 a k} \ln \frac{b+2 a w_{0}-k}{0.01 b-0.01 k} .
$$

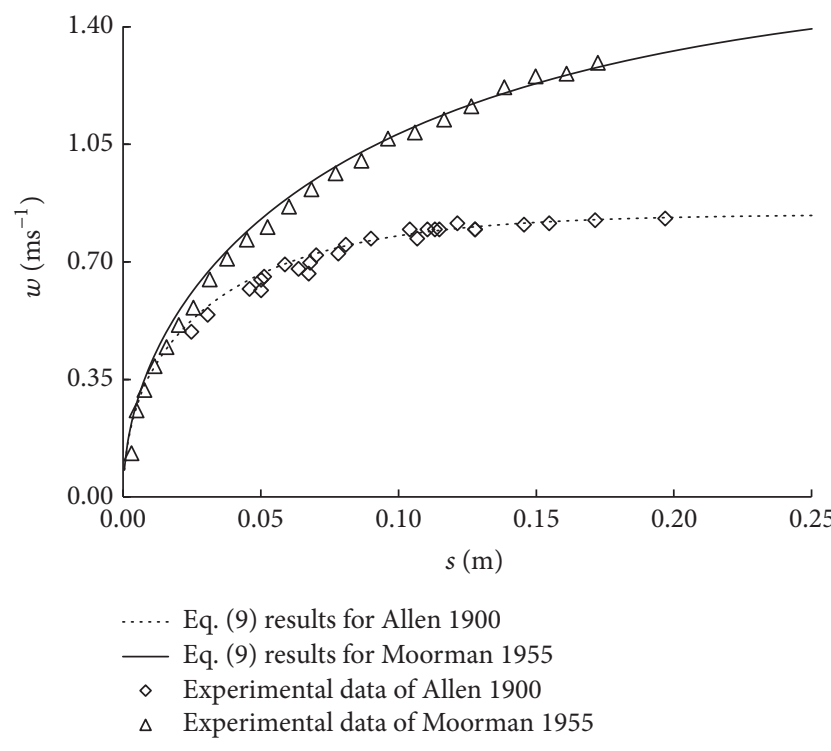

FIgURE 1: Fall velocities and distance relationships for experimental data and computational results.

For $w_{0}>1.01 w_{T}$ scenarios, the acceleration fall process was assumed to be completed when the fall velocity reached $101 \%$ of the terminal fall velocity. Applying (6) and $w=$ $1.01 w_{T}$ to (5) gives the corresponding time as follows:

$$
t_{L}=\frac{1}{k} \ln \frac{(2.01 k-0.01 b)\left(b+2 a w_{0}-k\right)}{(0.01 k-0.01 b)\left(b+2 a w_{0}+k\right)}+t_{0} .
$$

Substituting (12) into (8) gives

$$
\begin{aligned}
s_{L}= & \frac{k+b}{2 a} \ln \frac{b+2 a w_{0}+k}{2.01 k-0.01 b} \\
& +\frac{k-b}{2 a k} \ln \frac{b+2 a w_{0}-k}{0.01 k-0.01 b} .
\end{aligned}
$$

Note that the scenarios of $0.99 w_{T} \leqslant w_{0} \leqslant w_{T}$ or $w_{T} \leqslant$ $w_{0} \leqslant 1.01 w_{T}$ are not addressed in our study because they are very close to the terminal fall velocity.

\section{Validation by Experimental Data}

In order to validate (9), two data sets of a spherical particle acceleration falling through still fluid from Allen [31] and Moorman [32] were used. Allen [31] measured a steel sphere falling in a rectangular water tank; its depth, length, and width were $28 \mathrm{~cm}, 11.5 \mathrm{~cm}$, and $3 \mathrm{~cm}$, respectively. The water temperature was $17.8^{\circ} \mathrm{C}, \Delta=7.82, D=3.18 \mathrm{~mm}, w_{0}=0$, $w_{T}=83 \mathrm{~cm} / \mathrm{s}$, and the terminal Reynolds number $R_{T}=$ $w_{T} D / v=2440$. Moorman's (1955) tests were conducted in an oil tank with $D=12.7 \mathrm{~mm}, \rho_{s}=7780 \mathrm{~kg} / \mathrm{m}^{3}, \rho=876 \mathrm{~kg} / \mathrm{m}^{3}$, $v=3.54 \times 10^{-5} \mathrm{~m}^{2} \mathrm{~s}^{-1}, w_{0}=0, w_{T}=174.8 \mathrm{~cm} / \mathrm{s}$, and $R_{T}=627$. The fall velocities and distances of the experiments were plotted, and their comparisons with (9) were shown in Figure 1. Note that $\alpha=0$ and $\beta=24$ for $R<0.1, \alpha=0.5$ and $\beta=25.507$ for $0.1<R<1000, C_{A}=0.5$ for $R<1000$, and 


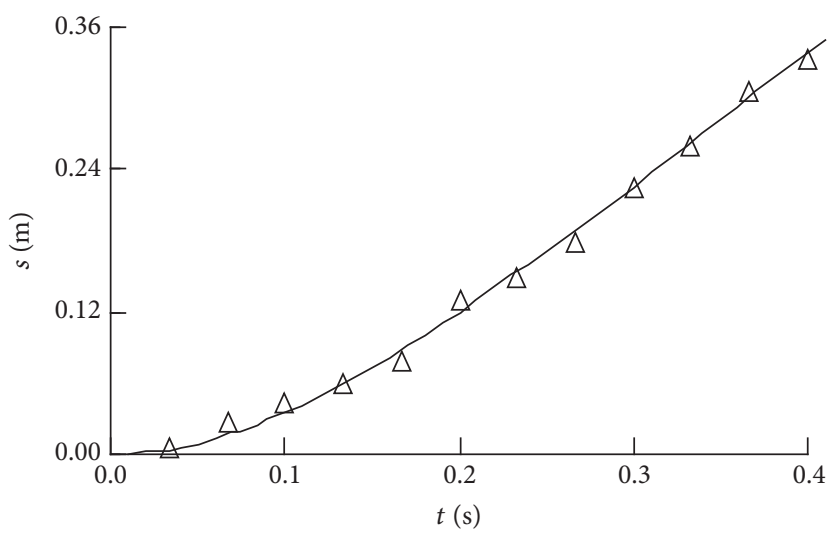

$\triangle$ Experimental data $\sim$ Eq. (8)

(a) Scenario 1



$\triangle$ Experimental data $\sim$ Eq. (8)

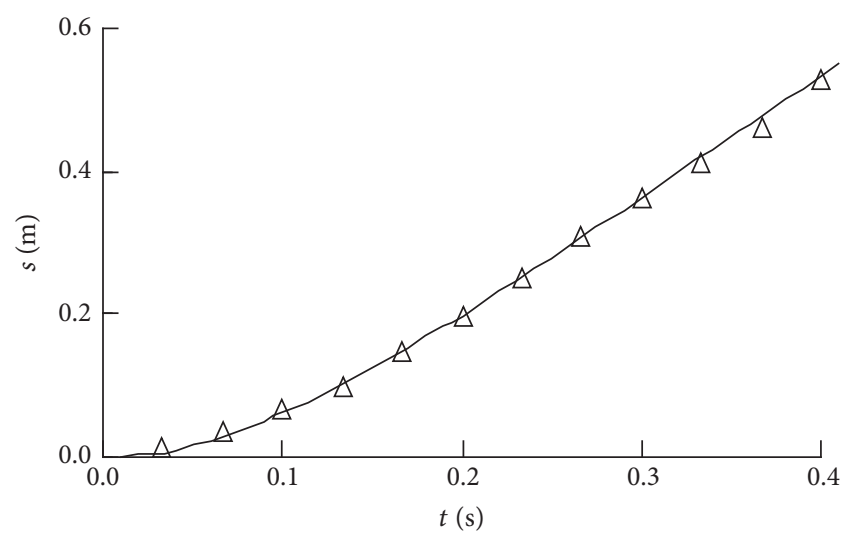

$\triangle$ Experimental data

$\longrightarrow$ Eq. (8)

(b) Scenario 2

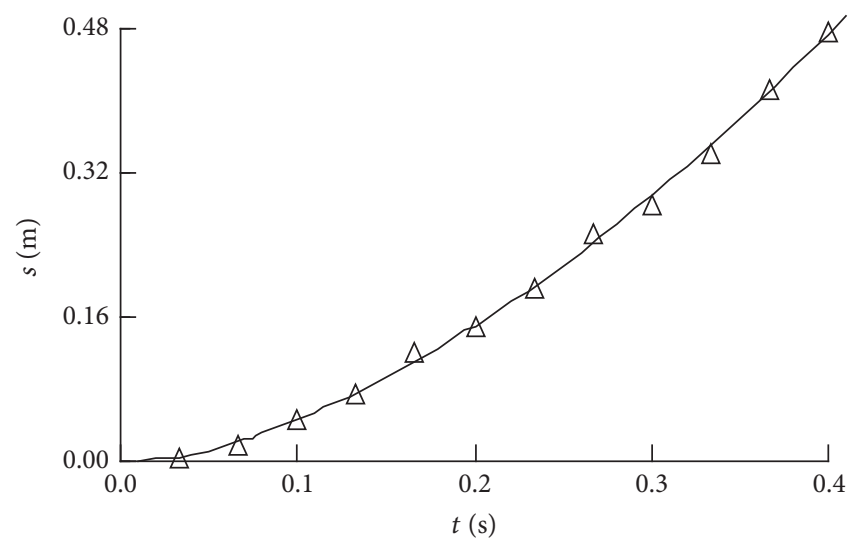

$\triangle$ Experimental data

$\triangle$ Eq. (8)

(c) Scenario 3

(d) Scenario 4

FIGURE 2: Fall distance relationship with time for $w_{0}=0$.

$\alpha=0.39, \beta=24$, and $C_{A}=2$ for $1000<R<10^{5}$ as reported by Guo [13] because of its good validation with Allen's [31] and Moorman's [32] test data. Figure 1 shows that the results from (9) for Allen's [31] scenario and the experimental data agreed well. As can be seen from Figure 1, the results from (9) are slightly overestimated according to the experiment with Moorman's scenarios [32].

In order to further validate (8), a series of experiments was conducted in the Hydraulic Lab at Ocean University of China. A rectangular glass tank was used with a height of $2.0 \mathrm{~m}$, a width of $0.3 \mathrm{~m}$, and a length of $0.3 \mathrm{~m}$. It was filled with tap water to a water depth of $1.8 \mathrm{~m}$. The water temperature was measured with a thermometer to be $20.7^{\circ} \mathrm{C}$. The spherical particles were made of plastic or glass. The diameters of the spherical particles were measured by Vernier calipers, and the mass of the spherical particles was measured by a balance scale. As a result, the densities of the spherical particles were calculated. The scale paper was pasted on the outside of the tank to obtain its distance. The spherical particle was released on the surface of the water. A video camera was used to record
TABLE 1: Experimental scenarios.

\begin{tabular}{lccc}
\hline Scenario number & $D(\mathrm{~mm})$ & $\rho_{s}\left(\mathrm{kgm}^{-3}\right)$ & $R_{T}$ \\
\hline 1 & 4.5 & 1125.6 & 520 \\
2 & 5.1 & 1236.7 & 880 \\
3 & 5.3 & 1445.6 & 1458 \\
4 & 14.4 & 1165 & 4020 \\
5 & 15.7 & 1165 & 4586 \\
6 & 16.6 & 1165 & 4982 \\
7 & 17.5 & 1165 & 5391 \\
\hline
\end{tabular}

the falling process at a speed of 30 frames per second. Video player software was used to read the video frame by frame, and the corresponding time and distance of each frame were obtained. Table 1 presents the detailed scenarios of the fall experiments.

Figure 2 illustrates the relationship between fall distance and time for scenarios 1 to 4 with $w_{0}=0$. Note that the fall 
in a laminar zone was not measured because of the limitation of camera performance and its very small value. Figures 2(a) and 2(b) show the data only in the transitional zone, and the horizontal coordinates of $t=0.113 \mathrm{~s}$ and $t=0.074 \mathrm{~s}$ are the critical values between transitional and turbulent zones as shown in Figures 2(c) and 2(d), respectively. It was observed that results from (8) agree well with experimental data, confirming that the determination of $C_{A}, \alpha$, and $\beta$ is reliable to compute the falling motion.

\section{Different Initial Velocity Effects on Total Fall Time and Distance}

Previous research shows that the initial velocity plays an important role in the fall time and distance [12, 13, 31, 32], and it deserves detailed comments. In terms of $w_{0}$ and $R_{T}$, the total fall time $T$ and the total acceleration fall distance $s_{T}$ were discussed in the following nine scenarios.

4.1. $w_{0} \leqslant w_{\text {crl }}$ for $R_{T}<0.1$. For $w_{0}<0.99 w_{T}$ scenarios, the fall velocity increases from $w_{0}$ to $w_{T}$. Therefore,

$$
\begin{gathered}
T=t_{S}\left(\alpha=0, \beta=24, C_{A}=0.5\right) \\
s_{T}=s_{S}\left(\alpha=0, \beta=24, C_{A}=0.5\right) .
\end{gathered}
$$

For $w_{0}>1.01 w_{T}$ scenarios, the fall velocity decreases from $w_{0}$ to $w_{T}$. Therefore,

$$
\begin{gathered}
T=t_{L}\left(\alpha=0, \beta=24, C_{A}=0.5\right) \\
s_{T}=s_{L}\left(\alpha=0, \beta=24, C_{A}=0.5\right) .
\end{gathered}
$$

4.2. $w_{\mathrm{cr} 1}<w_{0} \leqslant w_{\mathrm{cr} 2}$ for $R_{T}<0.1$. The fall velocity decreases from $w_{0}$ to $w_{\mathrm{cr} 1}$ and $w_{T}$, and the corresponding time increases from $t_{0}$ to $t_{\mathrm{cr} 1}$ and $t_{L}$. Therefore,

$$
\begin{aligned}
T= & \left(t_{\mathrm{cr} 1}-t_{0}\right)+\left(t_{L}-t_{\mathrm{cr} 1}\right) \\
s_{T}= & s\left(\alpha=0.5, \beta=25.057, C_{A}=0.5, t=t_{\mathrm{cr} 1}\right) \\
& \quad+s_{L}\left(\alpha=0, \beta=24, C_{A}=0.5, w_{0}=w_{\mathrm{cr} 1}\right),
\end{aligned}
$$

where $t_{L}$ can be obtained with $\alpha=0, \beta=24, C_{A}=0.5$, $w_{0}=w_{\mathrm{cr} 1}$, and $t_{0}=t_{\mathrm{cr} 1}$ using (12).

4.3. $w_{0}>w_{\mathrm{cr} 2}$ for $R_{T}<0.1$. The fall velocity decreases from $w_{0}$ to $w_{\mathrm{cr} 2}, w_{\mathrm{cr} 1}$, and $w_{T}$, and the corresponding time increases from $t_{0}$ to $t_{\mathrm{cr} 2}, t_{\mathrm{cr} 1}$, and $t_{L}$. Therefore,

$$
\begin{aligned}
T & =\left(t_{\mathrm{cr} 2}-t_{0}\right)+\left(t_{\mathrm{cr} 1}-t_{\mathrm{cr} 2}\right)+\left(t_{L}-t_{\mathrm{cr} 1}\right) \\
s_{T} & =s\left(\alpha=0.39, \beta=24, C_{A}=2, t=t_{\mathrm{cr} 2}\right)+s(\alpha \\
& =0.5, \beta=25.057, C_{A}=0.5, w_{0}=w_{\mathrm{cr} 2}, t_{0}=t_{\mathrm{cr} 2}, t \\
& \left.=t_{\mathrm{cr} 1}\right)+s_{L}\left(\alpha=0, \beta=24, C_{A}=0.5, w_{0}=w_{\mathrm{cr} 1}\right),
\end{aligned}
$$

where $t_{L}$ can be obtained with $\alpha=0, \beta=24, C_{A}=0.5$, $w_{0}=w_{\mathrm{cr} 1}$, and $t_{0}=t_{\mathrm{cr} 1}$ using (12).
4.4. $w_{0} \leqslant w_{\text {cr1 }}$ for $0.1<R_{T}<1000$. The fall velocity increases from $w_{0}$ to $w_{\mathrm{cr} 1}$ and $w_{T}$, and the corresponding time increases from $t_{0}$ to $t_{\mathrm{cr} 1}$ and $t_{S}$. Therefore,

$$
\begin{aligned}
T= & \left(t_{\mathrm{cr} 1}-t_{0}\right)+\left(t_{S}-t_{\mathrm{cr} 1}\right) \\
s_{T} & \\
= & s\left(\alpha=0, \beta=24, C_{A}=0.5, t=t_{\mathrm{cr} 1}\right) \\
& \quad+s_{S}\left(\alpha=0.5, \beta=25.057, C_{A}=0.5, w_{0}=w_{\mathrm{cr} 1}\right),
\end{aligned}
$$

where $t_{S}$ can be obtained with $\alpha=0.5, \beta=25.057, C_{A}=0.5$, $w_{0}=w_{\mathrm{cr} 1}$, and $t_{0}=t_{\mathrm{cr} 1}$ using $(10)$.

4.5. $w_{\text {cr } 1}<w_{0} \leqslant w_{\text {cr } 2}$ for $0.1<R_{T}<1000$. For $w_{0}<0.99 w_{T}$ scenarios, the fall velocity increases from $w_{0}$ to $w_{T}$. Therefore,

$$
\begin{gathered}
T=t_{S}\left(\alpha=0.5, \beta=25.057, C_{A}=0.5\right) \\
s_{T}=s_{S}\left(\alpha=0.5, \beta=25.057, C_{A}=0.5\right) .
\end{gathered}
$$

For $w_{0}>1.01 w_{T}$ scenarios, the fall velocity decreases from $w_{0}$ to $w_{T}$. Therefore,

$$
\begin{gathered}
T=t_{L}\left(\alpha=0.5, \beta=25.057, C_{A}=0.5\right) \\
s_{T}=s_{L}\left(\alpha=0.5, \beta=25.057, C_{A}=0.5\right) .
\end{gathered}
$$

4.6. $w_{0}>w_{\mathrm{cr} 2}$ for $0.1<R_{T}<1000$. The fall velocity decreases from $w_{0}$ to $w_{\mathrm{cr} 2}$ and $w_{T}$, and the corresponding time increases from $t_{0}$ to $t_{\mathrm{cr} 2}$ and $t_{L}$. Therefore,

$$
T=\left(t_{\mathrm{cr} 2}-t_{0}\right)+\left(t_{L}-t_{\mathrm{cr} 2}\right)
$$

$$
\begin{aligned}
& s_{T} \\
& \quad s\left(\alpha=0.39, \beta=24, C_{A}=2, t=t_{\mathrm{cr} 2}\right) \\
& \quad+s_{L}\left(\alpha=0.5, \beta=25.057, C_{A}=0.5, w_{0}=w_{\mathrm{cr} 2}\right),
\end{aligned}
$$

where $t_{L}$ can be obtained with $\alpha=0.5, \beta=25.057, C_{A}=0.5$, $w_{0}=w_{\mathrm{cr} 2}$, and $t_{0}=t_{\mathrm{cr} 2}$ using (12).

4.7. $w_{0} \leqslant w_{\text {crl }}$ for $R_{T}>1000$. The fall velocity increases from $w_{0}$ to $w_{\mathrm{cr} 1}, w_{\mathrm{cr} 2}$, and $w_{T}$, and the corresponding time increases from $t_{0}$ to $t_{\mathrm{cr} 1}, t_{\mathrm{cr} 2}$, and $t_{S}$. Therefore,

$$
\begin{aligned}
T & =\left(t_{\mathrm{cr} 1}-t_{0}\right)+\left(t_{\mathrm{cr} 2}-t_{\mathrm{cr} 1}\right)+\left(t_{s}-t_{\mathrm{cr} 2}\right) \\
s_{T} & =s\left(\alpha=0, \beta=24, C_{A}=0.5, t=t_{\mathrm{cr} 1}\right)+s(\alpha \\
& =0.5, \beta=25.057, C_{A}=0.5, w_{0}=w_{\mathrm{cr} 1}, t_{0}=t_{\mathrm{cr} 1}, t \\
& \left.=t_{\mathrm{cr} 2}\right)+s_{S}\left(\alpha=0.39, \beta=24, C_{A}=2, w_{0}=w_{\mathrm{cr} 2}\right),
\end{aligned}
$$

where $t_{S}$ can be obtained with $\alpha=0.39, \beta=24, C_{A}=2$, $w_{0}=w_{\mathrm{cr} 2}$, and $t_{0}=t_{\mathrm{cr} 2}$ using $(10)$.

Table 2 shows the total acceleration fall distance comparison between (21b) and experimental data with $w_{0}=0$. In Table $2, s_{T T}$ is the $s_{T}$ value of (21b), $s_{T E}$ is the $s_{T}$ value from the experiments, and the relative error was defined as 


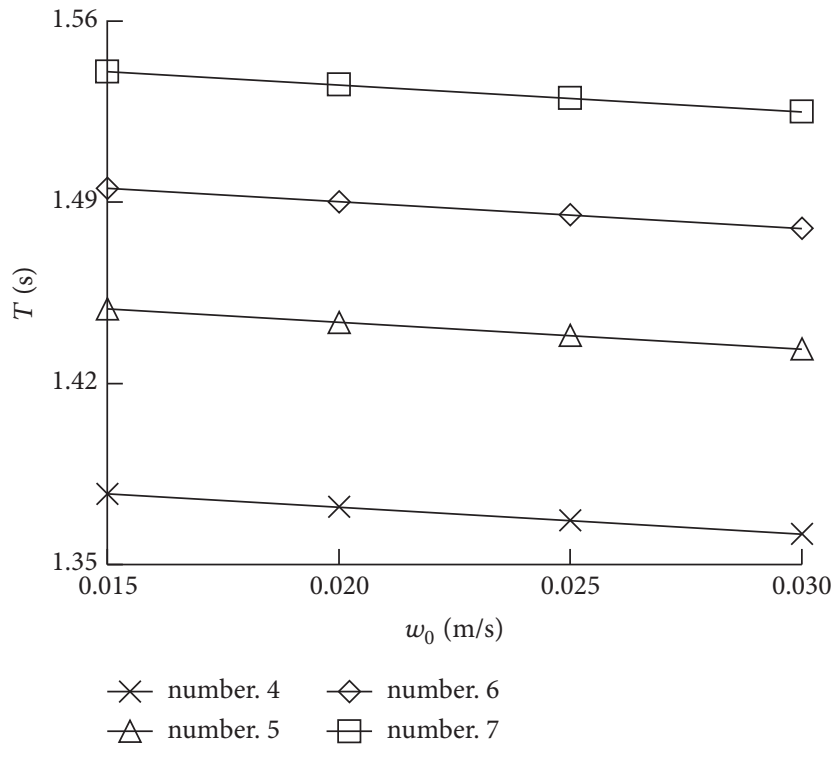

(a) $T$ and $w_{0}$



(b) $s_{T}$ and $w_{0}$

FIGURE 3: Relationship between $T, s_{T}$, and $w_{0}$ for $w_{\mathrm{cr} 1}<w_{0} \leqslant w_{\mathrm{cr} 2}$ and $R_{T}>1000$.

TABLE 2: Eq. (21b) and experimental distance of total acceleration with $w_{0}=0$.

\begin{tabular}{lccc}
\hline Scenario number & $S_{T T}(\mathrm{~m})$ & $S_{T E}(\mathrm{~m})$ & $E(\%)$ \\
\hline 4 & 0.299 & 0.290 & 3.1 \\
5 & 0.330 & 0.315 & 4.8 \\
6 & 0.347 & 0.357 & -2.8 \\
7 & 0.368 & 0.377 & -2.4 \\
\hline
\end{tabular}

$E=\left(\left(s_{T T}-s_{T E}\right) / s_{T E}\right) \times 100 \%$. It was found that the all of the relative errors between them were smaller than $5.0 \%$, confirming that (21b) is reliable to compute $s_{T}$ for $w_{0} \leqslant w_{\text {crl }}$ with $R_{T}>1000$.

4.8. $w_{\mathrm{cr} 1}<w_{0} \leqslant w_{\mathrm{cr} 2}$ for $R_{T}>1000$. The fall velocity increases from $w_{0}$ to $w_{\mathrm{cr} 2}$ and $w_{T}$, and the corresponding time increases from $t_{0}$ to $t_{\mathrm{cr} 2}$ and $t_{S}$. Therefore,

$$
\begin{aligned}
T= & \left(t_{\mathrm{cr} 2}-t_{0}\right)+\left(t_{S}-t_{\mathrm{cr} 2}\right) \\
s_{T}= & s\left(\alpha=0.5, \beta=25.057, C_{A}=0.5, t=t_{\mathrm{cr} 2}\right) \\
& +s_{S}\left(\alpha=0.39, \beta=24, C_{A}=2, w_{0}=w_{\mathrm{cr} 2}\right),
\end{aligned}
$$

where $t_{S}$ can be obtained with $\alpha=0.39, \beta=24, C_{A}=2$, $w_{0}=w_{\mathrm{cr} 2}$, and $t_{0}=t_{\mathrm{cr} 2}$ using (10).

Figure 3 shows the relationship between $T, s_{T}$, and $w_{0}$ for $w_{\mathrm{cr} 1}<w_{0} \leqslant w_{\mathrm{cr} 2}$ and $R_{T}>1000$. Note that $E_{V}$. is the abbreviation for the experimental values in Figures 3(b) and 4(b). Figure 3(a) illustrates that $T$ decreases with increasing $w_{0}$, and its reduction rate is higher for small $R_{T}$ than for large $R_{T}$. Figure $3(\mathrm{~b})$ shows that $s_{T}$ decreases slightly with increasing $w_{0}$, and its reduction rate increases very slightly with decreasing $R_{T}$, although it appears to remain constant to a great extent. It was also found that results from (22b) are consistent with the experimental values, as shown in Figure 3(b).

4.9. $w_{0}>w_{\mathrm{cr} 2}$ for $R_{T}>1000$. For $w_{0}<0.99 w_{T}$ scenarios, the fall velocity increases from $w_{0}$ to $w_{T}$. Therefore,

$$
\begin{gathered}
T=t_{S}\left(\alpha=0.39, \beta=24, C_{A}=2\right) \\
s_{T}=s_{S}\left(\alpha=0.39, \beta=24, C_{A}=2\right) .
\end{gathered}
$$

For $w_{0}>1.01 w_{T}$ scenarios, the fall velocity decreases from $w_{0}$ to $w_{T}$. Therefore,

$$
\begin{gathered}
T=t_{L}\left(\alpha=0.39, \beta=24, C_{A}=2\right) \\
s_{T}=s_{L}\left(\alpha=0.39, \beta=24, C_{A}=2\right) .
\end{gathered}
$$

For $w_{0}>w_{\mathrm{cr} 2}$ and $R_{T}>1000$ scenarios, the relationship between $T, s_{T}$, and $w_{0}$ is shown in Figure 4 . Figure 4 indicates that $T$ and $s_{T}$ increase with increasing $w_{0}$, and the increase rates are higher for small $R_{T}$ than for large $R_{T}$. Figure 4(b) shows that the results from (24b) agree with the experimental values. Consequently, the initial velocity plays a more important role in the acceleration motion for small $R_{T}$ than for large $R_{T}$ scenarios as shown in Figures 3 and 4 .

\section{Conclusions}

The acceleration motion of a spherical particle falling through still fluid was analytically and experimentally investigated using the BBO equation. The results of this study can be summarized as follows. 


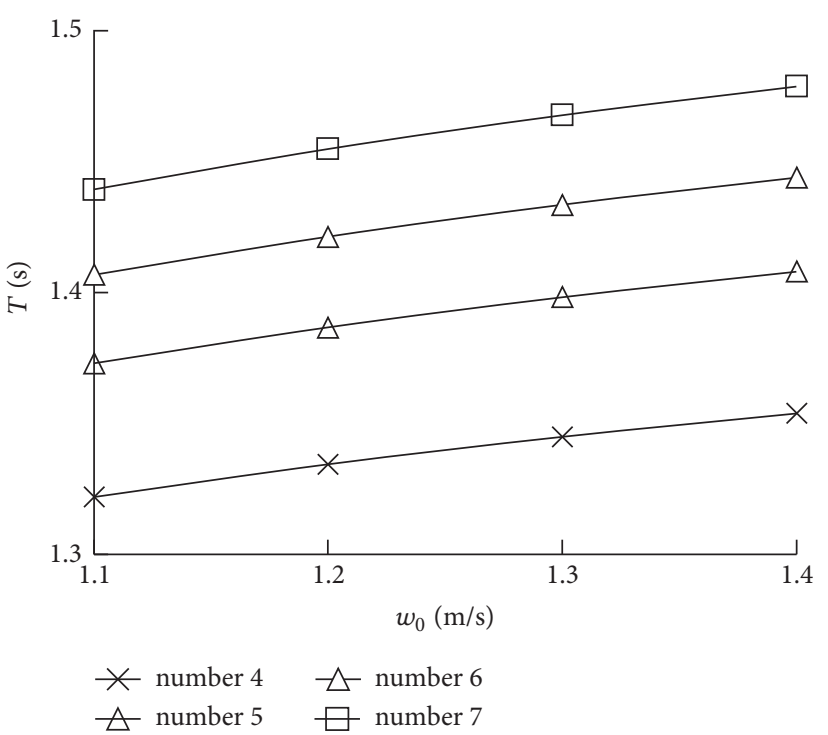

(a) $T$ and $w_{0}$

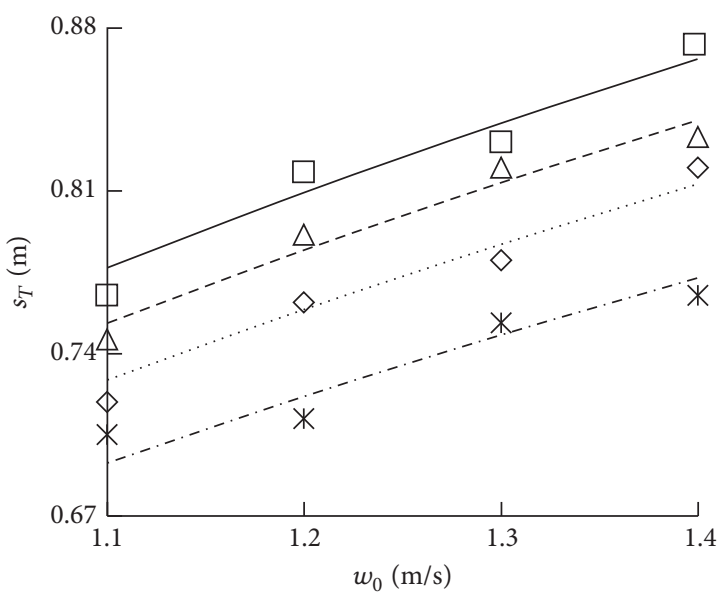

-.- Eq. (24b) for number $4 \quad \nVdash E_{V}$. for number. 4 Eq. (24b) for number $5 \diamond E_{V}$. for number. 5 - - - Eq. (24b) for number $6 \Delta E_{V}$. for number. 6 — Eq. (24b) for number $7 \quad \square E_{V}$. for number. 7

(b) $s_{T}$ and $w_{0}$

FIgURE 4: Relationship between $T, s_{T}$, and $w_{0}$ for $w_{0}>w_{\mathrm{cr} 2}$ and $R_{T}>1000$.

(1) The relationship between the drag coefficient and Reynolds number was studied, and different parameters in the drag coefficient equation were obtained with respect to the small, medium, and large Reynolds number zones. In terms of some assumptions, the finite fall time and distance equations were derived using the results reported by Guo [13].

(2) A simple experiment was conducted in order to measure a spherical particle fall process through still water. Literature and experimental data were used to validate the equations between fall time, velocity, and distance, confirming the determination of a drag force coefficient and an integrated added mass coefficient for different Reynolds number zones.

(3) The different initial velocities and terminal Reynolds numbers were taken into account, and the total fall time and distance equations were obtained. They fitted well with experimental data for $R_{T}>1000$ scenarios. It was found that the initial velocity plays a more important role in the falling motion for small $R_{T}$ than for large $R_{T}$ scenarios.

(4) The results of this study are possibly useful in hydraulic, environmental, and chemical engineering fields, such as particle size analysis, sediment deposition in water treatment engineering, and chemical reactions of particles in a fluidized bed.

It should be noted that this study experimentally measured fall data only in medium and large $R_{T}$ zones. We failed to measure the corresponding data in small $R_{T}$ zones because of camera performance limitations, spherical particle material, and spherical particle size; this might deserve further experimental evaluation in future work.

\section{Notations}

$a, b, c$ : Model parameters in (5) (-)

$C_{A}$ : Integrated added mass coefficient (-)

$C_{A 0}$ : Classic added mass coefficient (-)

$C_{B}: \quad$ Classic Basset force coefficient (-)

$C_{D}: \quad$ Drag coefficient (-)

$D: \quad$ Diameter of spherical particle $(\mathrm{m})$

g: $\quad$ Gravitational acceleration $\left(\mathrm{ms}^{-2}\right)$

$k$ : $\quad$ Interim parameter (-)

$m$ : $\quad$ Mass of fluid for the same volume of spherical particle $(\mathrm{kg})$

$m_{s}: \quad$ Spherical particle mass $(\mathrm{kg})$

$R: \quad$ Reynolds number $(-)$

$s: \quad$ Fall distance $(\mathrm{m})$

$t: \quad$ Time (s)

$t_{0}: \quad$ Initial time (s)

$w: \quad$ Fall velocity $\left(\mathrm{ms}^{-1}\right)$

$w_{0}: \quad$ Initial velocity $\left(\mathrm{ms}^{-1}\right)$

$w_{T}:$ Terminal fall velocity $\left(\mathrm{ms}^{-1}\right)$

$\alpha, \beta$ : Drag parameters in (2) (-)

$\Delta: \quad$ Ratio of sphere to fluid density (-)

$\mu$ : $\quad$ Dynamic viscosity of fluid $\left(\mathrm{Nsm}^{-2}\right)$

$\nu$ : $\quad$ Kinematic viscosity of fluid $\left(\mathrm{m}^{2} \mathrm{~s}^{-1}\right)$

$\rho: \quad$ Fluid density $\left(\mathrm{kgm}^{-3}\right)$

$\rho_{s}: \quad$ Density of a spherical particle $\left(\mathrm{kgm}^{-3}\right)$

$\tau$ : $\quad$ Dummy variable for integration $(-)$.

\section{Competing Interests}

The authors declare that they have no competing interests. 


\section{Acknowledgments}

This work was partly supported by the National Natural Science Foundation of China (nos. 51579229 and 51009123).

\section{References}

[1] G. G. Stokes, On the Effect of the Internal Friction of Fluids on the Motion of Pendulum, Pitt Press, 1851.

[2] C. W. Oseen, Über die Stoke'sche Formel und Über Eine Verwandte Aufgabe in der Hydrodynamik, Almqvist \& Wiksell, 1911.

[3] C. W. Oseen, Neuere Methoden und Ergebnisse in der Hydrodynamik, Akad, Verlagsgesellschaft, Leipzig, Germany, 1927.

[4] S. Kaplun and P. A. Lagerstrom, "Asymptotic expansions of Navier-Stokes solutions for small Reynolds numbers," Journal of Mathematics and Mechanics, vol. 6, no. 5, pp. 585-593, 1957.

[5] I. Proudman and J. R. A. Pearson, "Expansions at small Reynolds numbers for the flow past a sphere and a circular cylinder," Journal of Fluid Mechanics, vol. 2, no. 3, pp. 237-262, 1957.

[6] W. Chester, D. R. Breach, and I. Proudman, "On the flow past a sphere at low Reynolds number," Journal of Fluid Mechanics, vol. 37, no. 4, pp. 751-760, 1969.

[7] R. Clift, I. R. Grace, and M. E. Weber, "Bubbles, Drops, and Particles," Courier Corporation, 2005.

[8] S.-J. Liao, "An analytic approximation of the drag coefficient for the viscous flow past a sphere," International Journal of NonLinear Mechanics, vol. 37, no. 1, pp. 1-18, 2002.

[9] P. Y. Julien, Erosion and Sedimentation, Cambridge University Press, 2010.

[10] M. Hartman and J. G. Yates, "Free-fall of solid particles through fluids," Collection of Czechoslovak Chemical Communications, vol. 58, no. 5, pp. 961-982, 1993.

[11] T.-J. Chang and B. C. Yen, "Gravitational fall velocity of sphere in viscous fluid," Journal of Engineering Mechanics, vol. 124, no. 11, pp. 1193-1199, 1998.

[12] M. Jalaal, D. D. Ganji, and G. Ahmadi, "Analytical investigation on acceleration motion of a vertically falling spherical particle in incompressible Newtonian media," Advanced Powder Technology, vol. 21, no. 3, pp. 298-304, 2010.

[13] J. K. Guo, "Motion of spheres falling through fluids," Journal of Hydraulic Research, vol. 49, no. 1, pp. 32-41, 2011.

[14] Z.-G. Yin, Z.-L. Wang, L. Zhang, and S.-Q. Yin, "Theoretical analysis and experimental study of accelerating settlement of a spherical particle in still water with high Reynolds number," Advances in Science and Technology of Water Resources, vol. 36, no. 2, pp. 6-9, 2016 (Chinese).

[15] A. B. Basset, A Treatise on Hydrodynamics, Deighton Bell, Cambridge, UK, 1888.

[16] J. Boussinesq, Théorie Analytique de la Chaleur: Mise en Harmonie avec la Thermodynamique et avec la Théorie Mécanique de la Lumière, Gauthier-Villars, 1903.

[17] Y. Niño and M. García, "Using Lagrangian particle saltation observations for bedload sediment transport modelling," Hydrological Processes, vol. 12, no. 8, pp. 1197-1218, 1998.

[18] J. M. Ferreira, M. Duarte Naia, and R. P. Chhabra, "An analytical study of the transient motion of a dense rigid sphere in an incompressible Newtonian fluid," Chemical Engineering Communications, vol. 168, no. 1, pp. 45-58, 1998.

[19] W. W. Rubey, "Settling velocity of gravel, sand, and silt particles," American Journal of Science, vol. 25, no. 148, pp. 325-338, 1933.
[20] C. E. Lapple and C. B. Shepherd, "Calculation of particle trajectories," Industrial \& Engineering Chemistry, vol. 32, no. 5, pp. 605-617, 1940.

[21] C. E. Brennen, "A review of added mass and fluid inertial forces," Tech. Rep. CR 82.010, Naval Civil Engineering Laboratory, Port Hueneme, Calif, USA, 1982.

[22] W. S. Hamilton and J. E. Lindell, "Fluid force analysis and accelerating sphere tests," Journal of the Hydrualics Division, vol. 97, no. 6, pp. 805-817, 1971.

[23] F. Odar, "Verification of the proposed equation for calculation of the forces on a sphere accelerating in a viscous fluid," Journal of Fluid Mechanics, vol. 25, no. 3, pp. 591-592, 1966.

[24] E. E. Michaelides, "Review-the transient equation of motion for particles, bubbles, and droplets," Journal of Fluids Engineering, vol. 119, no. 2, pp. 233-247, 1997.

[25] M. van Aartrijk and H. J. H. Clercx, "Vertical dispersion of light inertial particles in stably stratified turbulence: the influence of the Basset force," Physics of Fluids, vol. 22, no. 1, 2010.

[26] C. J. Lawrence and S. Weinbaum, "The unsteady force on a body at low Reynolds number; the axisymmetric motion of a spheroid," Journal of Fluid Mechanics, vol. 189, pp. 463-489, 1988.

[27] Y. D. Sobral, T. F. Oliveira, and F. R. Cunha, "On the unsteady forces during the motion of a sedimenting particle," Powder Technology, vol. 178, no. 2, pp. 129-141, 2007.

[28] E. E. Michaelides, "A novel way of computing the Basset term in unsteady multiphase flow computations," Physics of Fluids A, vol. 4, no. 7, pp. 1579-1582, 1992.

[29] A. J. Dorgan and E. Loth, "Efficient calculation of the history force at finite Reynolds numbers," International Journal of Multiphase Flow, vol. 33, no. 8, pp. 833-848, 2007.

[30] F. A. Bombardelli, A. E. González, and Y. I. Niño, "Computation of the particle basset force with a fractional-derivative approach," Journal of Hydraulic Engineering, vol. 134, no. 10, pp. 1513-1520, 2008

[31] H. Allen, "The motion of a sphere in a viscous fluid," Philosophical Magazine, vol. 50, no. 306, pp. 519-534, 1900.

[32] R. W. Moorman, Motion of a spherical particle in the acceleration portion of free fall [Ph.D. dissertation], University of Iowa, Iowa City, Iowa, USA, 1955. 


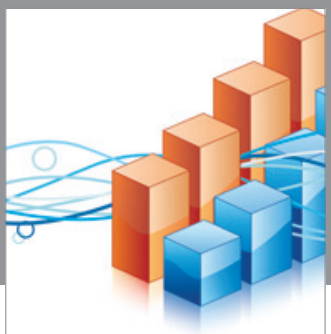

Advances in

Operations Research

vatem alat4

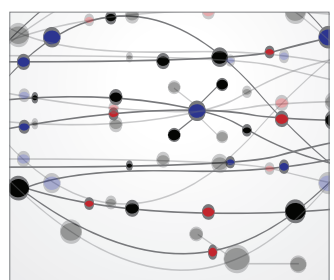

\section{The Scientific} World Journal
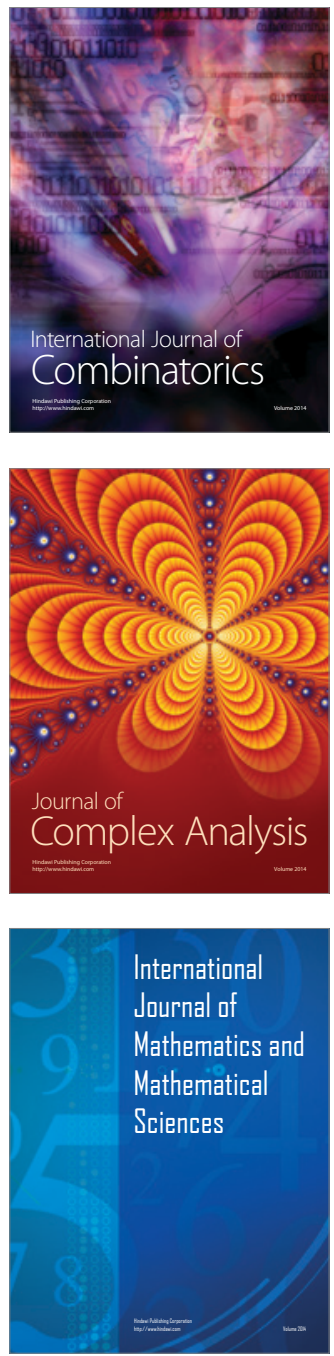
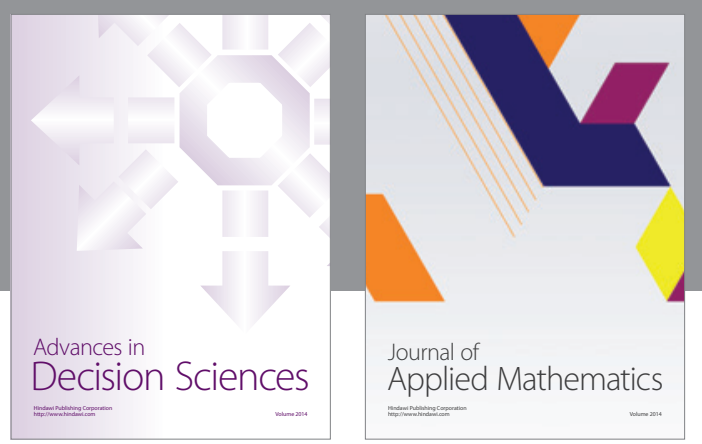

Algebra

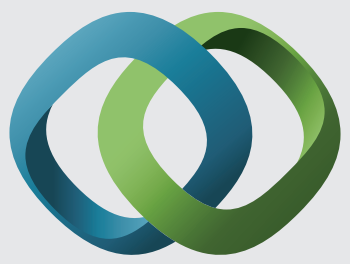

\section{Hindawi}

Submit your manuscripts at

https://www.hindawi.com
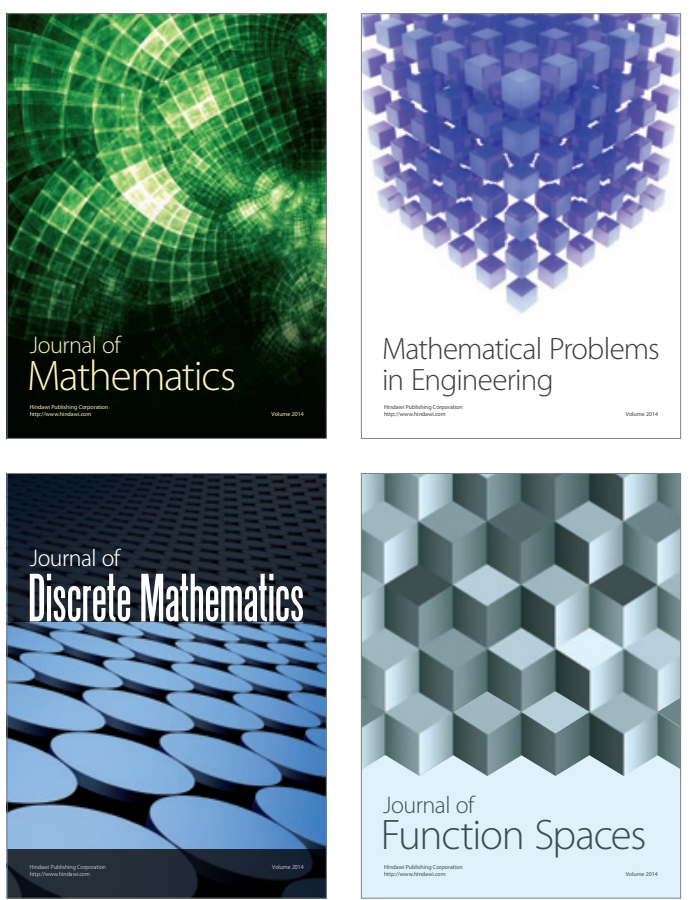

Mathematical Problems in Engineering
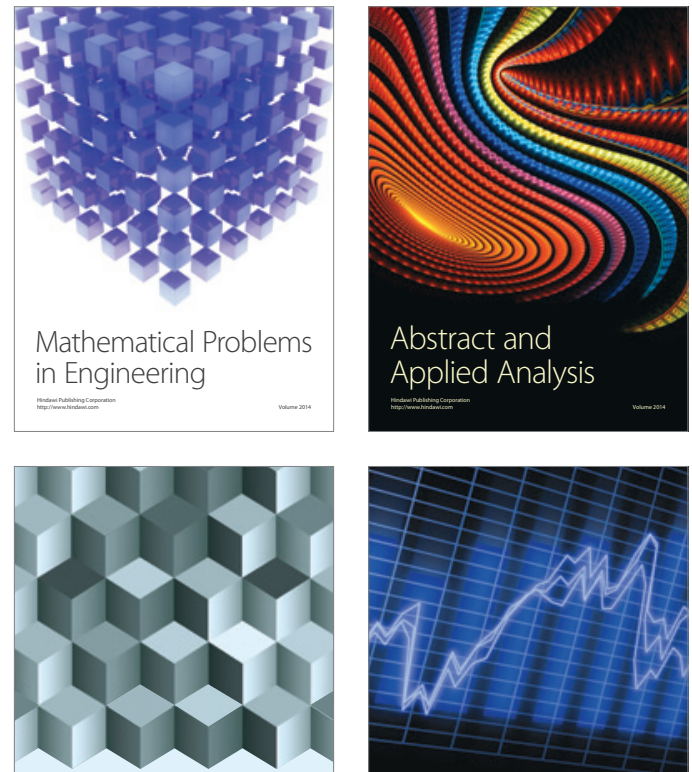

Journal of

Function Spaces

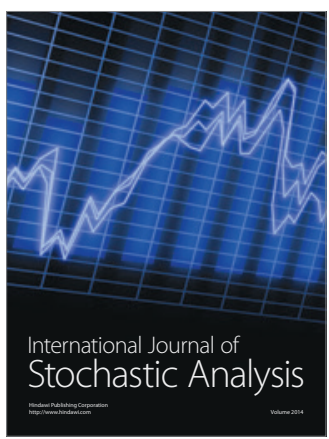

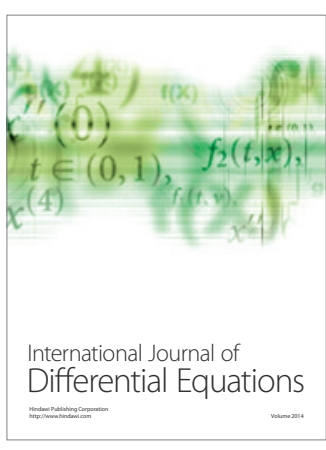
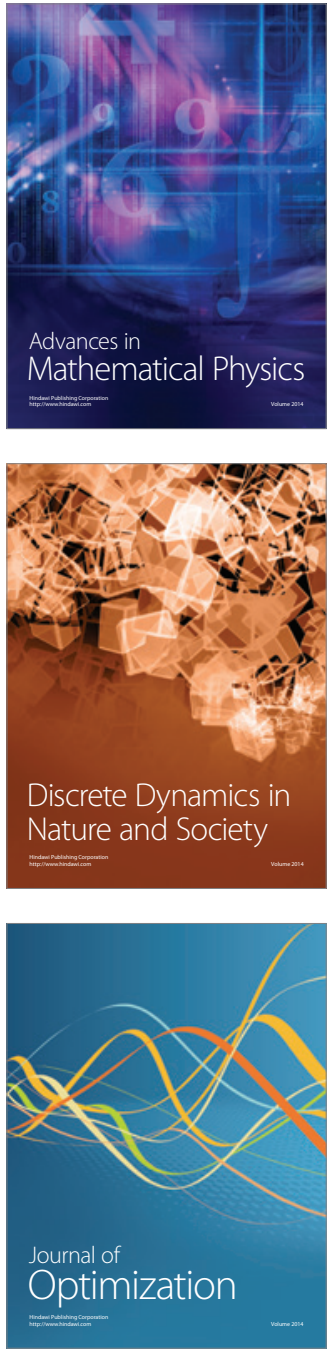